Annales Academiæ Scientiarum Fennicæ

Series A. I. Mathematica

Volumen 15, 1990, 381-399

\title{
REMOVABILITY THEOREMS FOR QUASIREGULAR MAPPINGS
}

\author{
P. Koskela and O. Martio
}

\section{Introduction}

A continuous mapping $f$ of an open set $G \subset \mathbf{R}^{n}$ into $\mathbf{R}^{n}$ is called $K$ quasiregular, $K \geq 1$, if $f$ is $\mathrm{ACL}^{n}$, i.e. the coordinate functions of $f$ belong to the local Sobolev space $\operatorname{loc} W_{n}^{1}(G)$, and if

$$
\left|f^{\prime}(x)\right|^{n} \leq K J(x, f)
$$

a.e. in $G$. Here $f^{\prime}(x)$ is the (formal) derivative of $f$ at $x, J(x, f)$ is the jacobian determinant of the matrix $f^{\prime}(x)$ and $\left|f^{\prime}(x)\right|$ stands for the supremum norm of the linear mapping $f^{\prime}(x): \mathbf{R}^{n} \rightarrow \mathbf{R}^{n}$. For $n=2$ and $K=1$ these mappings reduce to the class of analytic functions in $G$. Quasiregular mappings seem to form a proper generalization of analytic functions to higher dimensional euclidean spaces. For the theory of quasiregular mappings we refer to [MRV1-2] and [R].

Suppose that $C$ is a relatively closed subset of $G$. The removability theorem [CL, p. 5] of Painlevé and Besicovitch says that if the one dimensional Hausdorff measure $\mathcal{H}^{1}(C)$ of $C$ vanishes and if $f: G \backslash C \rightarrow \mathbf{R}^{2}$ is a bounded analytic function, then $f$ extends to an analytic function of $G$. For general quasiregular mappings $f: G \backslash C \rightarrow \mathbf{R}^{n}$ the following, much weaker result was proved in [MRV2]:

1.2. Theorem. Suppose that $C$ is of zero $n$-capacity. Then every bounded $K$-quasiregular mapping $f: G \backslash C \rightarrow \mathbf{R}^{n}$ extends to a $K$-quasiregular mapping of $G$.

The proof for this result has potential theoretic character: For bounded harmonic functions in the plane a set of zero 2-capacity is removable and there is a similar result in $\mathbf{R}^{n}, n \geq 2$, for coordinate functions of a quasiregular mapping $f$, see $[\mathrm{HKM}]$.

Rather precise removability theorems can be obtained in the plane.

1.3. Theorem. Suppose that $\mathcal{H}^{\lambda}(C)=0$ for all $\lambda>0$, i.e. the Hausdorff dimension $\operatorname{dim}_{\mathcal{H}}$ of $C$ is zero. Then every bounded plane $K$-quasiregular mapping $f: G \backslash C \rightarrow \mathbf{R}^{2}$ has a $K$-quasiregular extension $f^{*}$ to $G$. 
The proof uses the representation theorem [LV, p. 247] for plane quasiregular mappings: $f=g \circ h$ where $h$ is a quasiconformal mapping, i.e. a quasiregular homeomorphism, and $g$ is analytic. Since $\mathcal{H}^{1}(C)=0, h$ extends to a quasiconformal mapping $h^{*}$ of $G$, see [LV, p. 206]. A $K$-quasiconformal mapping is locally Hölder continuous with exponent $1 / K$. Thus $\operatorname{dim}_{\mathcal{H}}(C)=0$ implies $\operatorname{dim}_{\mathcal{H}}\left(h^{*}(C)\right)=0$. Hence the aforementioned theorem of Painlevé and Besicovitch shows that $g$ has an analytic extension $g^{*}$. Now $f^{*}=g^{*} \circ h^{*}$ is the required extension of $f$.

A look at the above proof gives the following result.

1.4. Theorem. Suppose that $f: G \backslash C \rightarrow \mathbf{R}^{2}$ is a bounded $K$-quasiregular mapping. If $\mathcal{H}^{1 / K}(C)=0$, then $f$ extends to a $K$-quasiregular mapping of $G$.

For $n \geq 3$ no results like Theorem 1.3 or 1.4 are known, except possibly for $K$ near 1 . The method of the proof certainly fails.

Theorem 1.2 has a remarkable extension, see [MRV2]: It holds if the mapping $f$ omits a set of positive $n$-capacity - of course, the extended mapping may now take the value $\infty$. The proof for this result employs the geometric theory of quasiregular mappings - modulus and capacity estimates.

The purpose of this paper is twofold. We prove a removability theorem, Theorem 4.1, for general quasiregular mappings $f: G \backslash C \rightarrow \mathbf{R}^{n}$ which omit a set of positive $n$-capacity. Our assumptions allow the set $C$ to be of positive $n$-capacity although $C$ is quite thin, for example $\operatorname{dim}_{\mathcal{H}} C=0$. The proof employs the geometric theory as in [MRV2]. We first show that $f$ can be extended to a continuous mapping $f^{*}$ of $G$. Thus we are naturally led to study removability questions for continuous mappings $f: G \rightarrow \mathbf{R}^{n}$ which are quasiregular in $G \backslash C$ this is done in Chapter 3. In Chapter 2 we introduce the conditions for the set $C$ used in the main theorem.

For locally Hölder continuous functions $f: G \rightarrow \mathbf{R}^{2}$ analytic in $G \backslash C$ the removability of $C$ is determined in terms of the Hölder exponent and the Hausdorff dimension of $C$. The following, very precise, result is due to L. Carleson, see e.g. [G, p.78]: If $\mathcal{H}^{\lambda}(C)=0$ and if $f$ is locally Hölder continuous in $G$ with exponent $\alpha \geq \lambda-1$ and analytic in $G \backslash C$, then $f$ extends to an analytic function of $G$. Reasoning as for Theorem 1.3 we obtain

1.5. Theorem. Suppose that $f: G \rightarrow \mathbf{R}^{2}$ is locally Hölder continuous with exponent $0<\alpha \leq 1$ and $K$-quasiregular in $G \backslash C$. If $\mathcal{H}^{\lambda}(C)=0, \lambda=$ $\min \left(1,1 / K+\alpha / K^{2}\right)$, then $f$ extends to a $K$-quasiregular mapping of $G$.

For $n \geq 2$ a different technique produces results which, in general, are better than Theorem 1.5. It suffices to assume that $\lambda \leq \min \{1, \alpha / n\}$ and hence $\lambda$ can be chosen independently of $K$, see Theorem 3.9. A careful analysis of a special semilocal Hölder class leads to results which allow removable sets $C$ with $\operatorname{dim}_{\mathcal{H}}(C)>1$. Such a result is Theorem 3.17 where the Minkowski dimension of $C$ and the Hölder exponent of $f$ are related. 
1.6. Remark. For each $\varepsilon>0$ there is a Cantor set $C \subset \mathbf{R}^{2}$ with $\operatorname{dim}_{\mathcal{H}}(C)<$ $\varepsilon$ and a $K$-quasiregular mapping $f$ of $\mathbf{R}^{2} \backslash C$ that is locally Hölder continuous in $\mathbf{R}^{2}$ with some exponent $\alpha>0$ but $f$ fails to extend to a quasiregular mapping of $\mathbf{R}^{2}$. The mapping $f$ can be constructed by composing a quasiconformal mapping as in [GV, Theorem 5] with an appropriate analytic function. Of course, $K$ and $\alpha$ depend on $\varepsilon$.

Since this work was completed, we have become aware of three other manuscripts dealing with removability questions for quasiregular mappings. T. Iwaniec and G.J. Martin [IM] have proved that for each $K$ and each $n$ there is a $\lambda=\lambda(K, n)>0$ such that closed sets $F$ of the even dimensional space $\mathbf{R}^{2 n}$ with $\mathcal{H}^{\lambda}(F)=0$ are removable for bounded $K$-quasiregular mappings of $\mathbf{R}^{2 n}$. Furthermore, P. Järvi and M. Vuorinen [JV] have established that certain selfsimilar Cantor sets are removable for quasiregular mappings omitting a finite but sufficiently large number of points. Finally, S. Rickman [Ri] has constructed examples of non-removable Cantor sets for bounded quasiregular mappings in $\mathbf{R}^{3}$.

\section{Modulus conditions}

We consider two modulus conditions, the $M$-condition and the $U M$-condition. The first was introduced in [M1] and further studied in [MS].

Let $G$ be an open set in $\mathbf{R}^{n}$ and $C$ a relatively closed subset of $G$. We say that a point $x_{0} \in G$ satisfies the $M$-condition with respect to $C$ if there exists a non-degenerate continuum $K \subset G$ such that

$$
\begin{gathered}
\left(K \backslash\left\{x_{0}\right\}\right) \cap C=\emptyset, \quad x_{0} \in K, \quad \text { and } \\
M\left(\Delta\left(K, C \cup \partial G ; G \backslash\left\{x_{0}\right\}\right)\right)<\infty .
\end{gathered}
$$

Here $\Delta(E, F ; A)$ stands for the family of all paths which join $E$ to $F$ in $A$ and $M(\Gamma)$ is the $n$-modulus of the path family $\Gamma$, see [V]. Note that in (2.2) we can write $\mathbf{R}^{n} \backslash\left\{x_{0}\right\}$ instead of $G \backslash\left\{x_{0}\right\}$ as well- $G \backslash\left\{x_{0}\right\}$ instead of $G$ is just used to avoid constant paths.

Clearly every point $x_{0} \in G \backslash C$ satisfies the $M$-condition with respect to $C$. Hence only points $x_{0} \in C$ are of interest in the $M$-condition. The $M$-condition seems also to depend on the domain $G$. However, writing $A=\mathbf{R}^{n} \backslash B^{n}\left(x_{0}, r_{0}\right)$, $x_{0} \in G$, we see that

$$
M_{0}=M\left(\Delta\left(K, A ; \mathbf{R}^{n}\right)\right) \leq \omega_{n-1}\left(\log \frac{r_{0}}{\delta}\right)^{1-n}<\infty
$$

whenever $K \subset G$ is a continuum with

$$
\delta=\operatorname{dia}(K)<r_{0}
$$


and with $x_{0} \in K$. Since

$$
\begin{aligned}
& M\left(\Delta\left(K, C \cup \partial G ; G \backslash\left\{x_{0}\right\}\right)\right) \\
& \quad \leq M_{0}+M\left(\Delta\left(K,(C \cup \partial G) \backslash A ; G \backslash\left\{x_{0}\right\}\right)\right),
\end{aligned}
$$

the points in $\partial G \cup C$ of distance $\varepsilon>0$ from $x_{0} \in G$ have no effect on the $M$-condition. Especially for points $x_{0} \in C$ the $M$-condition is independent of $G$.

We say that $C$ satisfies the $M$-condition (with respect to $G$ ) if each $x_{0} \in C$ satisfies the $M$-condition.

Next we say that $C$ satisfies the $U M$-condition (with respect to $G$ ) if for each compact set $F \subset G$ and for each $\varepsilon>0$ there is $\delta>0$ such that for every $x_{0} \in F$ there exists a continuum $K \subset G$ with the property (2.1) and

$$
\begin{gathered}
\operatorname{dia}(K) \geq \delta, \\
M\left(\Delta\left(K, C \cup \partial G ; G \backslash\left\{x_{0}\right\}\right)\right) \leq \varepsilon .
\end{gathered}
$$

If $C$ satisfies the $U M$-condition, then $C$ clearly satisfies the $M$-condition. The $U M$-condition ( $U M=$ uniform modulus) is a locally uniform version of the $M$ condition.

We shall frequently employ the following lemma which is a modification of a similar result in [M1] and [MS]. Note that the lemma will mostly be used for $C \cup \partial G$ instead of $C$.

2.5. Lemma. Let $C$ be a closed set in $\mathbf{R}^{n}, x_{0} \in \mathbf{R}^{n}$ and $K$ a continuum such that $x_{0} \in K$ and $K \backslash\left\{x_{0}\right\} \subset \mathbf{R}^{n} \backslash C$. There are constants $\beta>0$ and $b<\infty$ depending only on $n$ such that if

$$
m=M\left(\Delta\left(K, C ; \mathbf{R}^{n} \backslash\left\{x_{0}\right\}\right)\right) \leq \beta
$$

then there are radii $r_{i} \in\left(\operatorname{dia}(K) / 2^{i+1}, \operatorname{dia}(K) / 2^{i}\right), i=1,2, \ldots$, with

$$
S^{n-1}\left(x_{0}, r_{i}\right) \subset \mathbf{R}^{n} \backslash C,
$$

and

$$
M\left(\Delta\left(K^{\prime}, C ; \mathbf{R}^{n} \backslash\left\{x_{0}\right\}\right)\right) \leq b m,
$$

where $K^{\prime}=K \cup \cup_{i} S^{n-1}\left(x_{0}, r_{i}\right)$. 
Proof. Let $t_{i}=\operatorname{dia}(K) / 2^{i}, i=1,2, \ldots$, and $A_{0}=\mathbf{R}^{n} \backslash \bar{B}^{n}\left(x_{0}, t_{2}\right), A_{i}=$ $B^{n}\left(x_{0}, t_{i}\right) \backslash \bar{B}^{n}\left(x_{0}, t_{i+3}\right), i=1,2, \ldots$ Write

$$
\Gamma_{i}=\Delta\left(K, C ; A_{i}\right)
$$

First we prove that

$$
\sum_{i=0}^{\infty} M\left(\Gamma_{i}\right) \leq 3 M(\Gamma)
$$

here $\Gamma=\Delta\left(K, C ; \mathbf{R}^{n} \backslash\left\{x_{0}\right\}\right)$.

To this end, let $\varrho$ be an admissible function for $M(\Gamma)$. Now $\varrho$ is admissible for each $M\left(\Gamma_{i}\right)$ and no point $x \in \mathbf{R}^{n}$ belongs to more than three of the sets $A_{i}$; hence

$$
\sum_{i=0}^{\infty} M\left(\Gamma_{i}\right) \leq \sum_{i=0}^{\infty} \int_{A_{i}} \varrho^{n} d m \leq 3 \int_{\mathbf{R}^{n}} \varrho^{n} d m .
$$

The inequality (2.8) follows.

Observe that by [GM2, 2.18] and [HK, 2.6]

$$
M\left(\Gamma_{i}\right)=\operatorname{cap}\left(E_{i}\right)
$$

where $E_{i}=\left(C \cap \bar{A}_{i}, K \cap \bar{A}_{i} ; A_{i}\right)$ is a condenser whose capacity is defined as

$$
\operatorname{cap} E_{i}=\inf \int_{A}|\nabla u|^{n} d m
$$

here the infimum is taken over all functions $u \in C^{1}\left(A_{i}\right)$, continuous in $A_{i} \cup\left(\partial A_{i} \cap\right.$ $(C \cup K))$ with $u\left|K \cap \bar{A}_{i} \geq 1, u\right| C \cap \bar{A}_{i} \leq 0$.

Next for each $i=0,1, \ldots$ choose an admissible function for cap $E_{i}$ such that

$$
\int_{A_{i}}\left|\nabla u_{i}\right|^{n} d m \leq \operatorname{cap} E_{i}+M(\Gamma) / 2^{i+1}
$$

note that we may assume $M(\Gamma)>0$ since otherwise $C$ is of zero capacity and the existence of the required radii $r_{i}, i=1,2, \ldots$, follows easily. Consider the open sets

$$
\mathcal{U}_{i}=\left\{x \in A_{i}: u_{i}(x)>1 / 2\right\}, \quad i=1,2, \ldots
$$

If $\mathcal{U}_{i}$ does not contain any $S^{n-1}\left(x_{0}, r\right), r \in\left(t_{i+1}, t_{i}\right)$, then each such $S^{n-1}\left(x_{0}, r\right)$ meets both $A_{i} \backslash \mathcal{U}_{i}$ and $K$ yielding by [V, 10.12]

$$
\begin{aligned}
& \operatorname{cap}\left(\overline{A_{i} \backslash \mathcal{U}_{i}}, K \cap \bar{A}_{i} ; A_{i}\right) \\
& \quad=M\left(\Delta\left(\overline{A_{i} \backslash \mathcal{U}_{i}}, K \cap \bar{A}_{i} ; A_{i}\right)\right) \geq b_{1} \log 2
\end{aligned}
$$


where $b_{1}$ depends only on $n$. Next, observe that

$$
\begin{aligned}
\operatorname{cap}( & \left.\overline{A_{i} \backslash \mathcal{U}_{i}}, K \cap \bar{A}_{i} ; A_{i}\right) \leq 2^{n} \int_{A_{i}}\left|\nabla u_{i}\right|^{n} d m \\
& \leq 2^{n}\left(\operatorname{cap} E_{i}+M(\Gamma) / 2^{i+1}\right)=2^{n}\left(M\left(\Gamma_{i}\right)+M(\Gamma) / 2^{i+1}\right) \\
& \leq 2^{n} M(\Gamma),
\end{aligned}
$$

where we have used the definition of $\Gamma_{i}$ and (2.9)-(2.10). Now (2.11) and (2.12) yield a contradiction provided that

$$
M(\Gamma) \leq \beta=b_{1} 2^{-n-2} \log 2 .
$$

We have shown the existence of $S^{n-1}\left(x_{0}, r_{i}\right) \subset \mathcal{U} \subset \mathbf{R}^{n} \backslash C, t_{i+1}<r_{i}<t_{i}$.

It remains to prove that

$$
M\left(\Gamma^{\prime}\right) \leq b m,
$$

where $\Gamma^{\prime}=\Delta\left(K^{\prime}, C ; \mathbf{R}^{n} \backslash\left\{x_{0}\right\}\right)$ and $K^{\prime}=K \cup \cup_{i=1}^{\infty} S^{n-1}\left(x_{0}, r_{i}\right)$.

To this end, write $B_{0}=\mathbf{R}^{n} \backslash \bar{B}^{n}\left(x_{0}, r_{1}\right), B_{i}=B^{n}\left(x_{0}, r_{i}\right) \backslash \bar{B}^{n}\left(x_{0}, r_{i+1}\right)$, $i=1,2, \ldots$, and let $\Gamma_{i}^{\prime}=\Delta\left(K^{\prime} \cap \bar{B}_{i}, C \cap \bar{B}_{i} ; B_{i}\right), i=0,1,2, \ldots$. Since each $\gamma \in \Gamma^{\prime}$ has a subpath lying in some $\Gamma_{i}^{\prime}$ we conclude that

$$
M\left(\Gamma^{\prime}\right) \leq \sum_{i=0}^{\infty} M\left(\Gamma_{i}^{\prime}\right)
$$

Now we estimate $M\left(\Gamma_{i}^{\prime}\right)$.

For $i=0,1, \ldots$ the function $2\left|\nabla u_{i}\right|$ is admissible for $M\left(\Gamma_{i}^{\prime}\right)$. Thus by (2.9) and $(2.10)$

$$
\begin{aligned}
M\left(\Gamma_{i}^{\prime}\right) & \leq 2^{n} \int_{B_{i}}\left|\nabla u_{i}\right|^{n} d m \leq 2^{n} \int_{A_{i}}\left|\nabla u_{i}\right|^{n} d m \\
& \leq 2^{n}\left(\operatorname{cap} E_{i}+M(\Gamma) / 2^{i+1}\right) .
\end{aligned}
$$

Since $M\left(\Gamma_{i}\right)=\operatorname{cap} E_{i},(2.14)$ and (2.15) together with (2.8) yield

$$
\begin{aligned}
M\left(\Gamma^{\prime}\right) & \leq \sum_{i=0}^{\infty} M\left(\Gamma_{i}^{\prime}\right) \leq 2^{n}\left(\sum_{i=0}^{\infty} M\left(\Gamma_{i}\right)+M(\Gamma) / 2^{i+1}\right) \\
& \leq 2^{n+3} M(\Gamma) .
\end{aligned}
$$

The claim follows with $\beta=b_{1} 2^{-n-2} \log 2$ and $b=2^{n+3}$.

Next we produce a useful characterization for the $U M$-condition. 
2.16. Lemma. Suppose that $C$ is a relatively closed subset of an open set $G$. Then $C$ satisfies the $U M$-condition if and only if for every $x_{0} \in G$ and every $\varepsilon>0$ there is a neighborhood $\mathcal{U}$ of $x_{0}$ such that for each pair of points $x_{1}, x_{2} \in \mathcal{U}$ there is a continuum $K=K_{x_{1} x_{2}}$ with the properties:

$$
x_{1}, x_{2} \in K, \quad K \backslash\left\{x_{1}, x_{2}\right\} \subset G \backslash C,
$$

and

$$
M\left(\Delta\left(K, C \cup \partial G ; G \backslash\left\{x_{1}, x_{2}\right\}\right)\right) \leq \varepsilon .
$$

Proof. It is immediate that the condition of the lemma implies the $U M$ condition. To prove the converse assume that $C$ satisfies the $U M$-condition. Let $x_{0} \in G$ and pick an $r>0$ such that $F=\bar{B}^{n}\left(x_{0}, r\right)$ is a compact subset of $G$. By the $U M$-condition for each $\varepsilon>0$ there is $\delta>0$ such that for each $x \in F$ there is a continuum $K=K_{x} \subset G$ with properties (2.1), (2.3), and

$$
M(\Delta(K, C \cup \partial G ; G \backslash\{x\})) \leq \frac{\varepsilon}{2 b} ;
$$

here $b$ is the constant of Lemma 2.5.

Next, fix $\varepsilon>0$. We may assume that $\varepsilon /(2 b) \leq \beta$, see Lemma 2.5. Write

$$
r_{0}=\min (r, \delta / 8)
$$

and $\mathcal{U}=B\left(x_{0}, r_{0}\right)$. Then $\mathcal{U}$ is a neighborhood of $x_{0}$. Let $x_{1}, x_{2} \in \mathcal{U}$ and pick continua $K_{1}=K_{x_{1}}, K_{2}=K_{x_{2}}$ as in (2.19). By Lemma 2.5 we may replace $K_{j}$ with continua $K_{j}^{\prime}$ containing the spheres $S^{n-1}\left(x_{j}, r_{i}^{j}\right), i=1,2, \ldots, j=1,2$; note that

$$
M\left(\Delta\left(K_{j}^{\prime}, C \cup \partial G ; \mathbf{R}^{n} \backslash\left\{x_{0}\right\}\right)\right) \leq \frac{b \varepsilon}{2 b}=\frac{\varepsilon}{2},
$$

cf. (2.7).

Since $\left|x_{1}-x_{2}\right|<\delta / 4$, the continua $K_{1}^{\prime}$ and $K_{2}^{\prime}$ meet each other. Hence $K=K_{1}^{\prime} \cup K_{2}^{\prime}$ is a continuum with property (2.17). Furthermore, by (2.20)

$$
\begin{aligned}
& M\left(\Delta\left(K, C \cup \partial G ; G \backslash\left\{x_{1}, x_{2}\right\}\right)\right) \\
& \quad \leq \sum_{j=1}^{2} M\left(\Delta\left(K_{j}^{\prime}, C \cup \partial G ; G \backslash\left\{x_{j}\right\}\right)\right) \leq \frac{1}{2} \varepsilon+\frac{1}{2} \varepsilon=\varepsilon .
\end{aligned}
$$

This is (2.18) and thus the continuum $K$ has the desired properties.

For $\alpha>0$ and $C \subset \mathbf{R}^{n}$ we let $\mathcal{H}^{\alpha}(C)$ denote the usual $\alpha$-dimensional (outer) Hausdorff measure of $C$. The Hausdorff dimension of $C$ is written as $\operatorname{dim}_{\mathcal{H}}(C)$. 
2.21. Lemma. Suppose that $C$ satisfies the $M$-condition. Then $\operatorname{dim}_{\mathcal{H}}(C)=$ 0 . In particular, $C$ is totally disconnected.

Proof. By [MS, 3.1] the $n$-capacity density of $C$ is $=0$ at each point $x \in$ $C$. By $[\mathrm{M} 2,3.8]$ this implies that $\mathcal{H}^{\alpha}(C)=0$ for every $\alpha>0$. Consequently $\operatorname{dim}_{\mathcal{H}}(C)=0$ as required.

2.22. Remarks. (a) If $C$ satisfies the $U M$-condition, then $C$ satisfies the $M$-condition as well. Hence Lemma 2.21 holds for sets $C$ satisfying the $U M$ condition.

(b) In [M2] a set $C$ satisfying the $M$-condition but of positive $n$-capacity was constructed. A closer look at the construction shows that $C$ also satisfies the $U M$-condition. Thus there exist sets satisfying the $U M$-condition with positive $n$-capacity.

\section{Continuous removability}

Throughout this chapter $G$ is a domain in $\mathbf{R}^{n}$ and $C$ is a closed (relative to $G$ ) subset of $G$. We are mainly interested in the following problem: Suppose that $f: G \rightarrow \mathbf{R}^{n}$ is continuous and quasiregular in $G \backslash C$. Under which conditions is $f$ quasiregular in $G$ ?

The most difficult part in proving removability theorems for quasiregular mappings $f: G \backslash C \rightarrow \mathbf{R}^{n}$ is to show that $f$ is $\mathrm{ACL}^{n}$ in $G$. In most cases the ACLproperty is trivial and hence it remains to show that $\left|f^{\prime}\right|$ belongs to $\operatorname{loc} L^{n}(G)$. This fact is demonstrated in our first lemma.

3.1. Lemma. Suppose that $\mathcal{H}^{n-1}(C)=0$ and that $f: G \backslash C \rightarrow \mathbf{R}^{n}$ is a $K$-quasiregular mapping. If each $x_{0} \in C$ has a neighborhood $\mathcal{U}$ such that

$$
\int_{\mathcal{U} \backslash C}\left|f^{\prime}\right|^{n} d m<\infty
$$

then $f$ extends to a $K$-quasiregular mapping $f^{*}: G \rightarrow \mathbf{R}^{n}$.

Proof. Let $x_{0} \in C$ and pick a neighborhood $\mathcal{U}$ of $x_{0}$ as above. Since $\mathcal{H}^{n-1}(C)=0, f$ is ACL in $\mathcal{U}$ and by $(3.2), f$ is $\mathrm{ACL}^{n}$ in $\mathcal{U}$. On the other hand, $\left|f^{\prime}(x)\right|^{n} \leq K J(x, f)$ a.e. in $\mathcal{U}$, and these conditions imply that $f$ has a continuous extension $f^{*}$ to $\mathcal{U}$, see for example [BI, 2.1, 5.2]. The lemma follows.

For the next lemma we recall that a mapping $f: G \rightarrow \mathbf{R}^{n}$ is light if $f^{-1}(y)$ is a totally disconnected set for each $y \in \mathbf{R}^{n}$.

3.3. Lemma. Let $f: G \rightarrow \mathbf{R}^{n}$ be continuous and light. If $f$ is $K$ quasiregular in $G \backslash C$ and $m(f(C))=0$, then each $x \in C$ has a neighborhood $\mathcal{U}$ with

$$
\int_{\mathcal{U} \backslash C}\left|f^{\prime}\right|^{n} d m<\infty
$$


Proof. Note first that $f$ is discrete, open, and sense-preserving in each component of $G \backslash C$-this follows from the quasiregularity, see [MRV1, 2.26], and the lightness of $f$.

Fix $x_{0} \in C$, and pick a domain $D$ such that $x_{0} \in D, \bar{D} \subset G$, and $f^{-1}\left(f\left(x_{0}\right)\right)$ $\cap \partial D=\emptyset$; this is possible because $f$ is light and hence $f^{-1}\left(f\left(x_{0}\right)\right)$ is of topological dimension zero. Let $V$ be the $f\left(x_{0}\right)$-component of $\mathbf{R}^{n} \backslash f(\partial D)$ and let $\mathcal{U}$ be the $x_{0}$-component of $f^{-1}(V)$. Then $\mathcal{U}$ is an open neighborhood of $x_{0}$. If $y \in V \backslash f(C)$, then

$$
N(y, f, \mathcal{U}) \leq \sum_{x \in f^{-1}(y) \cap \mathcal{U}} i(x, f)=\mu(y, f, \mathcal{U})
$$

by the properties of the topological index $\mu$, see [MRV1, p. 6 and p. 11] for the definitions of $N, i$, and $\mu$. Next observe that $f(\partial \mathcal{U}) \subset \partial V$, and hence $y$ and $f\left(x_{0}\right)$ belong to the same component of $\mathbf{R}^{n} \backslash f(\partial \mathcal{U})$. But this means that

$$
\mu(y, f, \mathcal{U})=\mu\left(f\left(x_{0}\right), f, \mathcal{U}\right)
$$

and hence by (3.4)

$$
N(y, f, \mathcal{U}) \leq \mu\left(f\left(x_{0}\right), f, \mathcal{U}\right)=m<\infty
$$

for all $y \in V \backslash f(C)$. Since $m(f(C))=0$, (3.5) holds for a.e. $y$ in $V$. On the other hand [MRV1, 2.14] yields

$$
\begin{aligned}
\int_{\mathcal{U} \backslash C}\left|f^{\prime}\right|^{n} d m & \leq K \int_{\mathcal{U} \backslash C} J(x, f) d m=K \int_{\mathbf{R}^{n}} N(y, f, \mathcal{U} \backslash C) d m \\
& \leq \int_{\mathbf{R}^{n}} N(y, f, \mathcal{U}) d m \\
& =\int_{V \backslash f(C)} N(y, f, \mathcal{U}) d m<\infty,
\end{aligned}
$$

where (3.5) is used at the last step.

A mapping $f: G \rightarrow \mathbf{R}^{n}$ is said to be locally Hölder continuous if each $x_{0} \in G$ has a neighborhood $\mathcal{U}$ such that for some constants $0<\alpha \leq 1$ and $M<\infty$

$$
|f(x)-f(y)| \leq M|x-y|^{\alpha} \quad \text { for all } x, y \in \mathcal{U} .
$$

Further, $f$ is said to be locally Hölder continuous with exponent $\alpha$ if the above constant $\alpha$ is independent of $x_{0}$.

3.6. Theorem. Suppose that $\mathcal{H}^{\lambda}(C)=0$ for some $\lambda, 0<\lambda \leq n-1$, and let $f: G \rightarrow \mathbf{R}^{n}$ be light and locally Hölder continuous with exponent $\alpha \geq \lambda / n$. If $f$ is $K$-quasiregular in $G \backslash C$, then $f$ is $K$-quasiregular in $G$. 
Proof. By Lemmas 3.1 and 3.3 it suffices to show that $m(f(C))=0$.

To this end, let $F$ be a compact subset of $C$. Since $f$ is locally Hölder continuous with exponent $\alpha$, there is a neighborhood $\mathcal{U}$ of $F$ such that

$$
|f(x)-f(y)| \leq M|x-y|^{\alpha}
$$

for all $x, y \in \mathcal{U}$, where $M$ is independent of $x$ and $y$.

Let $\varepsilon>0$. Since $\mathcal{H}^{\lambda}(F)=0$, there is a covering of $F$ by balls $B^{n}\left(x_{i}, r_{i}\right)$, $r_{i} \leq 1$, such that $B^{n}\left(x_{i}, r_{i}\right) \subset \mathcal{U}$ and

$$
\sum_{i=1}^{\infty} r_{i}^{\lambda}<\varepsilon .
$$

Now $f\left(B^{n}\left(x_{i}, r_{i}\right)\right), i=1,2, \ldots$, is a covering of $f(F)$ and hence

$$
\begin{aligned}
m(f(F)) & \leq \Omega_{n} \sum_{i=1}^{\infty} \operatorname{dia}\left(f\left(B^{n}\left(x_{i}, r_{i}\right)\right)\right)^{n} \\
& \leq \Omega_{n} M^{n} \sum_{i=1}^{\infty} \operatorname{dia}\left(B^{n}\left(x_{i}, r_{i}\right)\right)^{\alpha n} \leq \Omega_{n} M^{n} 2^{\alpha n} \sum_{i=1}^{\infty} r_{i}^{\lambda} \\
& \leq \Omega_{n} M^{n} 2^{\alpha n} \varepsilon
\end{aligned}
$$

here (3.7) and (3.8) are also used. Letting $\varepsilon \rightarrow 0$ we obtain $m(f(F))=0$. Thus $m(f(C))=0$ as desired.

3.9. Theorem. Suppose that $\mathcal{H}^{\lambda}(C)=0,0<\lambda \leq 1$, and that $f: G \rightarrow \mathbf{R}^{n}$ is locally Hölder continuous with exponent $\alpha \geq \lambda / n$. If $f$ is $K$-quasiregular in $G \backslash C$, then $f$ is $K$-quasiregular in $G$.

Proof. Since $\mathcal{H}^{1}(C)=0, G \backslash C$ is a domain. If $f \mid G \backslash C$ is constant, then the claim is clear. Otherwise $f \mid G \backslash C$ is discrete and since $\mathcal{H}^{1}(C)=0, C$ is totally disconnected. Hence $f$ is light. The proof now follows from Theorem 3.6.

3.10. Remarks (a) For large values of $K$ Theorem 3.9 is better than Theorem 1.5. Note that the inequality $\alpha \geq \lambda / n$ does not include $K$.

(b) Theorem 3.9 gives the following result: If $\operatorname{dim}_{\mathcal{H}}(C)=0$ and if $f: G \rightarrow \mathbf{R}^{n}$ is locally Hölder continuous in $G$ and $K$-quasiregular in $G \backslash C$, then $f$ is $K$ quasiregular in $G$.

The preceding results have their roots in [MRV2, 4.1]. Next we relax the Hölder continuity condition of Theorem 3.6 slightly. If $D$ is an open, proper subset of $\mathbf{R}^{n}$, we let $W=\{Q\}$ denote the Whitney decomposition of $D$ into cubes $Q$. This means that each $Q \in W$ is a closed cube whose edges are of 
length $2^{-i}$ for some integer $i$ and parallel to the axes and the diameter of $Q$ is approximately proportional to the distance $d\left(Q, \mathbf{R}^{n} \backslash D\right)$, more precisely

$$
\operatorname{dia}(Q) \leq d\left(Q, \mathbf{R}^{n} \backslash D\right) \leq 4 \operatorname{dia}(Q) .
$$

Moreover, the interiors of $Q$ are mutually disjoint and $\cup Q=D$. For the construction of a Whitney decomposition $W$ see [S, p. 16]. The Whitney decomposition $W$ of $D$ is not unique but this fact has no importance in the following.

Suppose that $f: D \rightarrow \mathbf{R}^{n}$ and $0<\alpha \leq 1$. We say that $f$ belongs to loc $\operatorname{Lip}_{\alpha}(D)$ if there is $M<\infty$ such that

$$
|f(x)-f(y)| \leq M|x-y|^{\alpha}
$$

for each $x, y \in Q$ and for each $Q \in W$ where $W=\{Q\}$ is a Whitney decomposition of $D$. For the properties of the class $\operatorname{loc}_{\operatorname{Lip}}(D)$ see [GM1]. Note that the class loc $\operatorname{Lip}_{\alpha}(D)$ is properly contained in the class of locally Hölder continuous mappings in $D$ with exponent $\alpha$.

Finally we recall the definition of the Minkowski dimension of a compact set $F \subset \mathbf{R}^{n}$. For $\lambda>0$ and $r>0$ write

$$
M_{r}^{\lambda}(F)=\inf \left\{k r^{\lambda}: F \subset \bigcup_{i=1}^{k} B^{n}\left(x_{i}, r\right)\right\}
$$

and let

$$
M^{\lambda}(F)=\limsup _{r \rightarrow 0} M_{r}^{\lambda}(F) .
$$

The Minkowski dimension of $F$ is then defined similarly to the Hausdorff dimension as

$$
\operatorname{dim}_{\mathcal{M}}(F)=\inf \left\{\lambda>0: M^{\lambda}(F)<\infty\right\} .
$$

Note that $\operatorname{dim}_{\mathcal{M}}(F) \geq \operatorname{dim}_{\mathcal{H}}(F)$-for the properties of $\operatorname{dim}_{\mathcal{M}}$ see e.g. [MV].

3.11. Lemma. Let $f: G \backslash C \rightarrow \mathbf{R}^{n}$ be $K$-quasiregular. Suppose that $x \in C$ has a neighborhood $\mathcal{U}$ such that $\operatorname{dim}_{\mathcal{M}}(C \cap \overline{\mathcal{U}})=\lambda<n$ and $f$ lies in loc $\operatorname{Lip}_{\alpha}(\mathcal{U} \backslash C), \alpha>\lambda / n$. Then there is a neighborhood $V$ of $x$ with

$$
\int_{V \backslash C}\left|f^{\prime}\right|^{n} d m<\infty
$$

Proof. We may assume that $\mathcal{U}=B^{n}(x, r)$ and that $\overline{\mathcal{U}} \subset G$. Write $F=C \cap \overline{\mathcal{U}}$, and let $W$ be the Whitney decomposition of $\mathcal{U} \backslash F$.

For each $Q \in W$ let $Q^{\prime}$ denote the cube with the same center as $Q$, sides parallel to those of $Q$ and edge length $\ell\left(Q^{\prime}\right)=(3 / 2) \ell(Q)$. Note that $Q^{\prime}$ is covered with cubes $\widetilde{Q} \in W$ satisfying $\widetilde{Q} \cap Q \neq \emptyset$ and that

$$
\frac{1}{4} \ell(Q) \leq \ell(\widetilde{Q}) \leq 4 \ell(Q)
$$


for each such cube $\widetilde{Q}$.

Next pick a constant $b_{2}=b_{2}(n)$ so that $Q^{\prime} \subset B^{n}(x, r)$ whenever $Q \in W$ satisfies $Q \cap B^{n}\left(x, r / b_{2}\right) \neq \emptyset$; this is possible by the properties of $W$ since $x \in F$. We complete the proof by showing that

$$
\int_{V \backslash C}\left|f^{\prime}\right|^{n} d m<\infty
$$

where $V=B^{n}\left(x, r / b_{2}\right)$. Clearly we may assume that $2 r \leq 1$.

Notice first that

$$
\int_{V \backslash C}\left|f^{\prime}\right|^{n} d m \leq \sum_{i=1}^{\infty} \sum_{j=1}^{N_{i}} \int_{Q_{i j}}\left|f^{\prime}\right|^{n} d m
$$

where each $Q_{i j} \in W$ satisfies $\ell\left(Q_{i j}\right)=2^{-i}, Q_{i j}^{\prime} \subset B^{n}(x, r) \backslash F \subset G \backslash C$, and $N_{i}$ is the number of the cubes $Q_{i j} \in W$ that intersect $V$. Since $Q_{i j}^{\prime} \subset G \backslash C$ and $f: G \backslash C \rightarrow \mathbf{R}^{n}$ is $K$-quasiregular, [GLM, Lemma 4.2], see also [BI, 6.1], yields

$$
\int_{Q_{i j}}\left|f^{\prime}\right|^{n} d m \leq b_{3} \max _{y \in Q_{i j}^{\prime}}\left|f(y)-f\left(y_{i j}\right)\right|^{n},
$$

where $b_{3}$ depends only on $K$ and $n$ and $y_{i j}$ is the center of $Q_{i j}$. Note that (3.13) follows from the standard estimate of [GLM, p. 54] since each coordinate function of $f-f\left(y_{i j}\right)$ is an $F$-extremal, cf. [GLM, p. 71], with an appropriate $F$ and $\operatorname{cap}_{n}\left(Q_{i j}\right.$, int $\left.Q_{i j}^{\prime}\right)=c_{n}$ where $c_{n}$ depends only on $n$-here cap refers to $_{n}$ the $n$-capacity.

Next, since $f \in \operatorname{loc} \operatorname{Lip}_{\alpha}(\mathcal{U} \backslash C)$, we obtain

$$
\left|f(y)-f\left(y_{i j}\right)\right| \leq 5 \sqrt{n} M 2^{-i \alpha}=b_{4} 2^{-i \alpha}
$$

for each $y \in Q_{i j}^{\prime}$; here we have used the fact that every $y \in Q_{i j}^{\prime} \backslash Q_{i j}$ is contained in a cube $Q \in W$ meeting $Q_{i j}$ and hence $\ell(Q) \leq 4 \ell\left(Q_{i j}\right)$. On the other hand, by $[\mathrm{MV}, 3.9]$

$$
N_{i} \leq b_{5} 2^{i \lambda_{1}}, \quad i=1,2, \ldots
$$

for any $\lambda_{1}>\lambda=\operatorname{dim}_{\mathcal{M}}(F)$ for some $b_{5}$ independent of $i$. Combining (3.12)(3.15) we obtain

$$
\int_{V \backslash C}\left|f^{\prime}\right|^{n} d m \leq b_{6} \sum_{i=1}^{\infty} 2^{i\left(\lambda_{1}-\alpha n\right)}
$$

where $b_{6}=b_{3} b_{4}^{n} b_{5}$. Since $\alpha>\lambda / n$, the claim follows. 
3.16. Theorem. Let $f: G \backslash C \rightarrow \mathbf{R}^{n}$ be $K$-quasiregular. Suppose that each $x \in C$ has a neighborhood $\mathcal{U}$ such that $\operatorname{dim}_{\mathcal{M}}(C \cap \overline{\mathcal{U}})=\lambda<n-1$ and $f$ lies in loc $\operatorname{Lip}_{\alpha}(\mathcal{U} \backslash C)$ for some $\alpha>\lambda / n$. Then $f$ extends to a $K$-quasiregular mapping $f^{*}: G \rightarrow \mathbf{R}^{n}$.

Proof. The claim follows from Lemmas 3.1 and 3.11.

3.17. Theorem. Suppose that $\operatorname{dim}_{\mathcal{M}}(F) \leq \lambda<n-1$ for each compact $F \subset C$ and that $f: G \rightarrow \mathbf{R}^{n}$ is locally Hölder continuous with exponent $\alpha>\lambda / n$. If $f$ is $K$-quasiregular in $G \backslash C$, then $f$ is $K$-quasiregular in $G$.

Proof. If $f$ is locally Hölder continuous with exponent $\alpha$ in $G$, then each $x \in$ $C$ has a neighborhood $\mathcal{U}$ such that $f \in \operatorname{loc} \operatorname{Lip}_{\alpha}(\mathcal{U} \backslash C)$. Since $\operatorname{dim}_{\mathcal{M}}(C \cap \overline{\mathcal{U}}) \leq \lambda$, the claim follows from Theorem 3.17.

3.18. Remark. It may happen that $\operatorname{dim}_{\mathcal{M}}(C)>\operatorname{dim}_{\mathcal{H}}(C)$, hence Theorem 3.17 does not imply Theorem 3.9. Note that there are countable closed sets $C$ with $\operatorname{dim}_{\mathcal{M}}(C)>0$.

\section{A removability theorem for quasiregular mappings}

Suppose that $G$ is a domain in $\mathbf{R}^{n}$ and $C$ is a relatively closed subset of $G$.

4.1. Theorem. Suppose that $f: G \backslash C \rightarrow \mathbf{R}^{n}$ is a $K$-quasiregular mapping omitting a set of positive $n$-capacity. If $C$ satisfies the $U M$-condition, then $f$ has a $K$-quasimeromorphic extension $f^{*}: G \rightarrow \mathbf{R}^{n} \cup\{\infty\}$.

The formulation of the theorem needs an explanation. First, the mapping $f^{*}$ may take the value $\infty$. Hence, as in the classical analytic plane case, we say that $f^{*}: G \rightarrow \mathbf{R}^{n} \cup\{\infty\}$ is $K$-quasimeromorphic if for each $x \in G$ either $f^{*}$ is $K$-quasiregular or, in the case $f^{*}(x)=\infty, g \circ f$ is $K$-quasiregular at a neighborhood of $x$; here $g$ is a sense-preserving Möbius transformation such that $g(\infty) \neq \infty$. Next let $F$ be a closed proper subset of $\mathbf{R}^{n}$. If $F_{1} \subset \mathbf{R}^{n} \backslash F$ is a nondegenerate continuum, then we write $\Gamma\left(F_{1}\right)=\Delta\left(F_{1}, F ; \mathbf{R}^{n}\right)$. Now $M\left(\Gamma\left(F_{1}\right)\right)>0$ or $M\left(\Gamma\left(F_{1}\right)\right)=0$ for each such continuum $F_{1}$. In the first case we say that $F$ is of positive $n$-capacity and write $\operatorname{cap}_{n} F>0$. In the second case $F$ is said to be of zero $n$-capacity; this we write $\operatorname{cap}_{n} F=0$. Since

$$
M\left(\Gamma\left(F_{1}\right)\right)=\operatorname{cap}_{n}\left(F_{1}, \mathbf{R}^{n} \backslash F\right),
$$

this definition agrees with the usual definition of a set of zero $n$-capacity, see $[\mathrm{MRV} 2$, p. 6] or [HKM].

Finally, note that if $f$ is bounded, then $f$ omits a set of positive $n$-capacity and the mapping $f^{*}$ in Theorem 4.1 is $K$-quasiregular.

To prove Theorem 4.1 we need three lemmas; we assume that $f: G \backslash C \rightarrow \mathbf{R}^{n}$ and $C$ satisfy the conditions of the theorem. 
4.2. Lemma. The mapping $f$ has a continuous extension $f^{*}: G \rightarrow \mathbf{R}^{n} \cup$ $\{\infty\}$.

Proof. We may assume that $f$ is non-constant. It suffices to show that $f$ can be extended continuously to each point $x_{0} \in C$. Fix $x_{0} \in C$ and let $\varepsilon>0$. Pick a neighborhood $\mathcal{U} \subset G$ of $x_{0}$ such that the conditions (2.17) and (2.18) of Lemma 2.16 hold. Let $x_{1}, x_{2} \in \mathcal{U} \backslash C$ and let $K_{x_{1} x_{2}}$ be a continuum with the properties in Lemma 2.16. Write $\Gamma$ for the family of paths joining $f\left(K_{x_{1} x_{2}}\right)$ to $\mathbf{R}^{n} \backslash f(G \backslash C)$ in $\mathbf{R}^{n} \cup\{\infty\}$; note that $f(G \backslash C)$ is an open subset of $\mathbf{R}^{n}$ because $f$ is open. Let $\Gamma^{*}$ be the family of maximal lifts (under $f$ ) of the paths in $\Gamma$ starting at $K_{x_{1} x_{2}}$, see [MRV3, 3.11]. Then each $\gamma^{*} \in \Gamma^{*}$ ends either in $C$ or in $\partial G \cup\{\infty\}$. By the fundamental modulus inequality for quasiregular mappings, see $[\mathrm{P}]$,

$$
M(\Gamma) \leq K^{n-1} M\left(\Gamma^{*}\right)
$$

note that the inner dilatation $K_{I}(f)$ of $f$ satisfies $K_{I}(f) \leq K^{n-1}$, see [MRV1, pp. 14-15]. On the other hand, condition (2.18) of Lemma 2.16 yields

$$
M\left(\Gamma^{*}\right) \leq \varepsilon .
$$

Thus (4.3) and (4.4) imply

$$
M(\Gamma) \leq K^{n-1} \varepsilon
$$

Next write $t=q\left(f\left(K_{x_{1} x_{2}}\right)\right)$-the spherical diameter of $f\left(K_{x_{1} x_{2}}\right)$, see [MRV2, 3.10]. Since $\operatorname{cap}_{n}\left(\mathbf{R}^{n} \backslash f(G \backslash C)\right)>0$, [MRV2, Lemma 3.1] together with (4.5) shows that $t \leq \delta$ where $\delta \rightarrow 0$ as $\varepsilon \rightarrow 0$. Since the spherical distance $q\left(f\left(x_{1}\right), f\left(x_{2}\right)\right)$ of $f\left(x_{1}\right)$ and $f\left(x_{2}\right)$ satisfies

$$
q\left(f\left(x_{1}\right), f\left(x_{2}\right)\right) \leq q\left(f\left(K_{x_{1} x_{2}}\right)\right) \leq \delta,
$$

the Cauchy criterion shows that $f$ has a continuous extension to $x_{0}$.

4.6. Remark. It was proved in [Vu] that if $f: G \backslash C \rightarrow \mathbf{R}^{n}$ is quasiregular and omits a set of positive $n$-capacity, then $f$ has a unique asymptotic limit at $x_{0} \in C$ provided that $x_{0}$ satisfies the $M$-condition with respect to $C$. Lemma 4.2 shows that a slightly stronger assumption yields a continuous extension.

4.7. Lemma. The mapping $f^{*}: G \rightarrow \mathbf{R}^{n} \cup\{\infty\}$ is either a constant or light and open.

Proof. Suppose that $f^{*}$ is not a constant. Fix $y \in \mathbf{R}^{n} \cup\{\infty\}$. Then

$$
f^{*-1}(y) \subset f^{-1}(y) \cup C
$$


and since $f^{-1}(y)$ is a discrete set of points in $G \backslash C$ and since $C$ is totally disconnected, see Lemma 2.21 and Remark $2.22(\mathrm{a}), f^{*-1}(y)$ is a subset of a totally disconnected set. Thus $f^{*}$ is light.

Next we show that $f^{*}$ is open. Note that $f$ is open at any $x_{0} \in G \backslash C$. Suppose that $x_{0} \in C$. Since $f^{*}$ is light and $C$ is totally disconnected, there are arbitrarily small connected neighborhoods $D \subset G$ of $x_{0}$ such that

$$
\partial D \subset G \backslash C
$$

and

$$
f^{*-1}\left(x_{0}\right) \cap \partial D=\emptyset .
$$

Fix such a domain $D$. It suffices to show that $f^{*}\left(x_{0}\right) \in \operatorname{int} f^{*}(D)$. Since $f^{*}$ is continuous, we may assume that $f^{*}\left(x_{0}\right) \neq \infty$ and that $f^{*}(\bar{D})$ is a compact subset of $\mathbf{R}^{n}$.

Denote the $f^{*}\left(x_{0}\right)$-component of $\mathbf{R}^{n} \backslash f(\partial D)$ by $D^{\prime}$, and let $V=D^{\prime} \backslash f^{*}(\bar{D})$. Since $f^{*}(\bar{D})$ is compact, $V$ is open. We shall show that $V=\emptyset$.

Suppose not. Pick a connected component $V^{\prime}$ of $V$. If $\partial V^{\prime} \cap D^{\prime}=\emptyset$, then $\partial V^{\prime} \subset \partial D^{\prime}$ and hence $V^{\prime}=D^{\prime}$ which is impossible because $f^{*}\left(x_{0}\right) \in D^{\prime} \backslash V^{\prime}$. Thus there is $y \in \partial V^{\prime} \cap D^{\prime}$. Now $y \in f^{*}(\bar{D}) \backslash f(\partial D)$, hence there is a point $x$ in $D$ with $f^{*}(x)=y$. Pick a continuum $K_{x}$ as in the $M$-condition for $x$ with $K_{x} \backslash\{x\} \subset D \backslash C$ and

$$
M\left(\Delta\left(K_{x}, C \cup \partial G ; G \backslash\{x\}\right)\right) \leq 1 .
$$

On the other hand, $y=f^{*}(x)$ is a boundary point of a domain $V^{\prime}$, hence for each $T>0$ there is a non-degenerate continuum $K^{\prime} \subset V^{\prime}$ such that

$$
M\left(\Delta\left(f^{*}\left(K_{x}\right), K^{\prime} ; \mathbf{R}^{n}\right)\right) \geq T
$$

note that $f^{*}\left(K_{x}\right)$ is a non-degenerate continuum containing the point $y$.

Next, write $\Gamma=\Delta\left(f^{*}\left(K_{x}\right), K^{\prime} ; \mathbf{R}^{n}\right)$, and let $\Gamma^{*}$ be the family of all maximal lifts (under $f \mid D \backslash C$ ) of $\Gamma$ starting at $K_{x} \backslash\{x\}$. Since $K^{\prime} \cap f^{*}(\bar{D})=\emptyset$, each $\gamma^{*} \in \Gamma^{*}$ ends either at $C \cap D$ or at $\partial D$. Thus

$$
\begin{aligned}
M\left(\Gamma^{*}\right) & \leq M\left(\Delta\left(K_{x}, C ; \mathbf{R}^{n} \backslash\{x\}\right)\right)+M\left(\Delta\left(K_{x}, \partial D ; \mathbf{R}^{n}\right)\right) \\
& \leq 1+M<\infty
\end{aligned}
$$

where $M$ is independent of $K^{\prime}$; note that $M<\infty$ because $K_{x}$ is a compact subset of $D$. Since $f$ is $K$-quasiregular, we conclude that

$$
M(\Gamma) \leq K^{n-1} M\left(\Gamma^{*}\right) \leq K^{n-1}(1+M) .
$$

Choosing $T$ in (4.10) large enough we obtain a contradiction. Hence $V=\emptyset$.

Now $D^{\prime} \backslash f^{*}(\bar{D})=V=\emptyset$ and thus $D^{\prime} \subset f^{*}(\bar{D})$. Since $D^{\prime}$ does not meet $f^{*}(\partial D), D^{\prime} \subset f^{*}(D)$ and since $D^{\prime}$ is an open neighborhood of $f^{*}\left(x_{0}\right)$ in $f^{*}(D)$, we have the desired conclusion $f^{*}\left(x_{0}\right) \in \operatorname{int} f^{*}(D)$. 
4.12. Lemma. The mapping $f^{*}$ is locally Hölder continuous in $G \backslash f^{*-1}(\infty)$.

Proof. Since $f: G \backslash C \rightarrow \mathbf{R}^{n}$ is locally Hölder continuous as a quasiregular mapping, see [MRV2, 3.2], it suffices to show that any $x_{0} \in C$ with $f^{*}\left(x_{0}\right) \neq \infty$ has a neighborhood $\mathcal{U}$ with $\left|f^{*}(x)-f^{*}(y)\right| \leq M|x-y|^{\alpha}$ for all $x, y \in \mathcal{U}$, where $\alpha>0$ and $M<\infty$ are independent of the points $x$ and $y$.

To this end, fix such a point $x_{0} \in C$ and pick a ball $B^{n}\left(x_{0}, 8 r\right) \subset G$ such that $\infty \notin f^{*}\left(\bar{B}^{n}\left(x_{0}, 6 r\right)\right)$ and for any $x \in B^{n}\left(x_{0}, r\right)$ there is a continuum $K_{x}$ with $x \in K_{x}, K_{x} \backslash\{x\} \subset G \backslash C, 8 r \leq \operatorname{dia}\left(K_{x}\right) \leq 9 r$, and $M\left(K, C \cup \partial G ; \mathbf{R}^{n} \backslash\{x\}\right) \leq \beta$, where $\beta$ is the constant of Lemma 2.5. This is possible because $C$ satisfies the $U M$-condition and $f^{*}$ is continuous. Let $x \in B^{n}\left(x_{0}, r\right)$ and pick a continuum $K_{x}$ as above. Let $r_{1}>r_{2}>\ldots$ be the sequence of radii given by Lemma 2.5. From this sequence we select every second and still denote this new sequence by $\left(r_{i}\right)$. Write

$$
L_{i}=\max _{y \in \bar{B}\left(x, r_{i}\right)}\left|f^{*}(y)-f^{*}(x)\right|, \quad i=1,2, \ldots
$$

Since $f^{*}$ is open by Lemma 4.7 ,

$$
L_{i}=\max _{y \in S^{n-1}\left(x, r_{i}\right)}\left|f(y)-f^{*}(x)\right| ;
$$

note that $S^{n-1}\left(x, r_{i}\right) \subset G \backslash C$.

For each $i=1,2, \ldots$ let $\Gamma_{i}$ be the family of paths which connect $f\left(S^{n-1}\left(x, r_{i+1}\right)\right)$ to $f\left(S^{n-1}\left(x, r_{i}\right)\right)$ in $\mathbf{R}^{n}$. Let $\Gamma_{i}^{*}$ be the family of maximal lifts under $f \mid B^{n}\left(x, r_{i}\right) \backslash C$ of $\Gamma_{i}$ starting at $S^{n-1}\left(x, r_{i+1}\right)$. Each path $\gamma^{*}$ in $\Gamma_{i}^{*}$ ends either in $C$ or in $S^{n-1}\left(x, r_{i}\right)$. Thus

$$
\begin{aligned}
M\left(\Gamma_{i}^{*}\right) \leq & M\left(\Delta\left(S^{n-1}\left(x, r_{i+1}\right), C ; \mathbf{R}^{n}\right)\right) \\
& +M\left(\Delta\left(S^{n-1}\left(x, r_{i+1}\right), S^{n-1}\left(x, r_{i}\right) ; \mathbf{R}^{n}\right)\right) \\
\leq & b \beta+\omega_{n-1}\left(\log r_{i} / r_{i+1}\right)^{1-n} \\
\leq & b \beta+\omega_{n-1}\left(\log \frac{\operatorname{dia}\left(K_{x}\right) / 2^{2 i+2}}{\operatorname{dia}\left(K_{x}\right) / 2^{2(i+1)+1}}\right)^{1-n} \\
= & b \beta+\omega_{n-1}(\log 2)^{1-n}=b_{1} ;
\end{aligned}
$$

here we used the fact that we had chosen every second of the original radii of Lemma 2.5. On the other hand, (4.15), [V, 6.4], and the quasiregularity of $f$ imply

$$
\begin{aligned}
M\left(\Gamma_{i}\right) \leq & M\left(\Delta \left(f\left(S^{n-1}\left(x, r_{i+1}\right)\right),\right.\right. \\
& \left.f\left(S^{n-1}\left(x, r_{i}\right) \cup \partial f(G \backslash C) ; f(G \backslash C)\right)\right) \\
\leq & K^{n-1} M\left(\Gamma_{i}^{*}\right) \leq K^{n-1} b_{1} .
\end{aligned}
$$


Let $y_{0} \in S^{n-1}\left(x, r_{i+1}\right)$ be such that $L_{i+1}=\left|f\left(y_{0}\right)-f(x)\right|$ and write $z_{0}=$ $L_{i}\left(f\left(y_{0}\right)-f(x)\right)+f(x)$. Since $f^{*}$ is open, for each $s \in\left(L_{i}-L_{i+1}, L_{i}\right)$ the sphere $S^{n-1}\left(z_{0}, s\right)$ meets both $f\left(S^{n-1}\left(x, r_{i}\right)\right)$ and $f\left(S^{n-1}\left(x, r_{i+1}\right)\right)$. Hence [V, 10.12] yields

$$
M\left(\Gamma_{i}\right) \geq b_{2} \log \frac{L_{i}}{L_{i}-L_{i+1}}
$$

Here $b_{2}$ depends only on $n$. Now (4.16) and (4.17) give

$$
L_{i+1} \leq b_{3} L_{i}, \quad i=1,2, \ldots,
$$

where $b_{3}=\left(e^{t}-1\right) / e^{t}, t=K^{n-1} b_{1} / b_{2}$, is independent of $x$ and $i$.

From (4.18) we obtain by iteration

$$
L_{i} \leq b_{3}^{i-1} L_{1}, \quad i=1,2, \ldots
$$

Finally, let $y \in B^{n}\left(x_{0}, r\right)$. Note that $r_{1}>\operatorname{dia}\left(K_{x}\right) / 4 \geq 2 r>|x-y|$; hence we may pick an integer $i$ such that

$$
r_{i+1} \leq|x-y|<r_{i} .
$$

Now (4.13) and (4.19) imply

$$
\left|f^{*}(x)-f^{*}(y)\right| \leq L_{i} \leq b_{3}^{i-1} L_{1} .
$$

On the other hand,

$$
|x-y| \geq r_{i+1} \geq \operatorname{dia}\left(K_{x}\right) / 2^{2 i+2} \geq 2 r / 2^{i} ;
$$

hence

$$
i \geq \log \left(\frac{2 r}{|x-y|}\right)^{1 /(2 \log 2)} .
$$

By $(4.20)$ this yields (observe that $b_{3}<1$ )

$$
\left|f^{*}(x)-f^{*}(y)\right| \leq M|x-y|^{\alpha},
$$

where $\alpha=-\log b_{3} / 2 \log 2>0$ and

$$
\begin{aligned}
M & =b_{3}^{-1} L_{1}(2 r)^{\left(\log b_{3}\right) /(2 \log 2)} \\
& \leq 2 b_{3}^{-1}(2 r)^{\left(\log b_{3}\right) /(2 \log 2)} \max _{z \in \bar{B}^{n}\left(x_{0}, 6 r\right)}|f(z)|<\infty
\end{aligned}
$$

are independent of $x$ and $y$. The lemma follows. 
Proof for Theorem 4.1. Since the definition of quasimeromorphic mappings is local, it suffices to show that any $x_{0} \in C$ has a neighborhood $\mathcal{U}$ with $f^{*} \mid \mathcal{U}$ $K$-quasimeromorphic; here $f^{*}$ is the mapping given by Lemma 4.2. Fix $x_{0} \in C$, and pick a sense-preserving Möbius transformation $g$ with $g(\infty) \neq \infty$. Assume first that $f^{*}(\infty) \neq \infty$; then Lemmas 4.2 and 4.12 imply that $f^{*}$ is locally Hölder continuous in a neighborhood $\mathcal{U}$ of $x_{0}$. Thus $f^{*}$ is $K$-quasiregular in $\mathcal{U}$ by Lemma 2.21 and Remark 3.10 (b).

Suppose finally that $f^{*}\left(x_{0}\right)=\infty$. Now $g \circ f^{*}$ is bounded in a neighborhood $\mathcal{U}$ of $x_{0}$ and $K$-quasiregular in $\mathcal{U} \backslash C$. Hence Lemma 4.2 yields a continuous extension $(g \circ f)^{*}: \mathcal{U} \rightarrow \mathbf{R}^{n} \cup\{\infty\}$. Moreover, $(g \circ f)^{*}=g \circ f^{*}$ and $(g \circ f)^{*}\left(x_{0}\right) \neq \infty$; hence the proof follows by applying the above reasoning to $(g \circ f)^{*}$.

\section{References}

[BI] BoJARski, B., and T. IwANIEC: Analytical foundations of quasiconformal mappings in $\mathbf{R}^{n}$. - Ann. Acad. Sci. Fenn. Ser. A I Math. 8, 1983, 257-324.

[CL] Collingwood, E.F., and A.J. Lohwater: The theory of cluster sets. - Cambridge University Press, Cambridge, 1966.

[G] Garnett, J.: Analytic capacity and measure. - Lecture Notes in Mathematics 297, Springer-Verlag, New York-Berlin-Heidelberg, 1972.

[GM1] Gehring, F.W., and O. Martio: Lipschitz classes and quasiconformal mappings. - Ann. Acad. Sci. Fenn. Ser. A I Math. 10, 1985, 203-219.

[GM2] Gehring, F.W., and O. Martio: Quasiextremal distance domains and extension of quasiconformal mappings. - J. Analyse Math. 45, 1985, 181-206.

[GV] GehrING, F.W., and J. V̈̈̈Ï̈̈L̈: Hausdorff dimension and quasiconformal mappings. J. London Math. Soc. 6, 1973, 504-512.

[GLM] Granlund, S., P. Lindqvist, and O. Martio: Conformally invariant variational integrals. - Trans. Amer. Math. Soc. 277, 1983, 43-73.

[HKM] Heinonen, J., T. Kilpeläinen, and O. Martio: Non-linear potential theory. - To appear

[HK] Herron, D.A., and P. Koskela: Quasiextremal distance domains and conformal mappings onto circle domains. - Complex Variables 15, 1990, 167-179.

[IM] Iwaniec, T., and G.J. Martin: Quasiregular mappings in even dimensions. - Preprint 19, Institut Mittag-Leffler, Stockholm, 1990.

[JV] J̈̈RVI, P., and M. Vuorinen: Self-similar Cantor sets and quasiregular mappings. Preprint 27, Institut Mittag-Leffler, Stockholm, 1990.

[LV] Lehto, O., and K.I. Virtanen: Quasiconformal mappings in plane. - Springer-Verlag, New York-Berlin-Heidelberg, 1973.

[M1] MarTio, O.: Equicontinuity theorem with an application to variational integrals. - Duke Math. J. 42, 1975, 569-581.

[M2] Martio, O.: Capacity and measure densities. - Ann. Acad. Sci. Fenn. Ser. A I Math. 4, 1979, 109-118.

[MRV1] Martio, O., S. RICKMAN, and J. VÄISÄLÄ: Definitions for quasiregular mappings. Ann. Acad. Sci. Fenn. Ser. A I Math. 448, 1969, 1-40.

[MRV2] Martio, O., S. RICKMAN, and J. VÄISÄLÄ: Distortion and singularities of quasiregular mappings. - Ann. Acad. Sci. Fenn. Ser. A I Math. 465, 1970, 1-13. 
[MRV3] Martio, O., S. Rickman, and J. VÄISÄLÄ: Topological and metric properties of quasiregular mappings. - Ann. Acad. Sci. Fenn. Ser. A I Math. 488, 1971, 1-31.

[MS] Martio, O., and J. Sarvas: Density conditions in the $n$-capacity. - Indiana Univ. Math. J. $26,1977,761-776$.

[MV] Martio, O., and M. Vuorinen: Whitney cubes, p-capacity, and Minkowski content. Exposition. Math. 5, 1987, 17-40.

[P] Poletskil , E.A.: The modulus method for non-homeomorphic quasiconformal mappings. - Math. Sb. 83, 1970, 261-272 (Russian).

[R] Reshetnyak, YU.G.: Space mappings with bounded distortion. - Translation of Mathematical Monographs 73, American Mathematical Society, Providence, 1989.

[Ri] Rickman, S.: Nonremovable Cantor sets for bounded quasiregular mappings. - To appear.

[S] SteIn, E.M.: Singular integrals and differentiability properties of functions. - Princeton University Press, Princeton, 1970.

[V] VÄISÄLÄ, J.: Lectures on $n$-dimensional quasiconformal mappings. - Lecture Notes in Mathematics 229, Springer-Verlag, New York-Berlin-Heidelberg, 1971.

[Vu] Vuorinen, M.: Exceptional sets and boundary behavior of quasiregular mappings in $n$ space. - Ann. Acad. Sci. Fenn. Ser. A I Math. Diss. 11, 1976, 1-44.

P. Koskela

University of Jyväskylä

Department of Mathematics

Seminaarinkatu 15

SF-40100 Jyväskylä

Finland
O. Martio

University of Jyväskylä

Department of Mathematics

Seminaarinkatu 15

SF-40100 Jyväskylä

Finland

Received 11 June 1990 